\title{
Nlrp3 Activation Induces Il-18 Synthesis and Affects the Epithelial Barrier Function in Reactive Cholangiocytes
}

Luca Maroni, ${ }^{*}$ Laura Agostinelli, Stefania Saccomanno, ${ }^{*}$ Claudio Pinto, ${ }^{*}$ Debora M. Giordano, ${ }^{*}$ Chiara Rychlicki, ${ }^{*}$

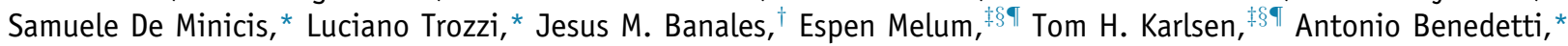
Gianluca Svegliati Baroni, ${ }^{*}$ and Marco Marzioni*

From the Department of Gastroenterology and Hepatology, * Università Politecnica delle Marche, Ancona, Italy; the Department of Liver and Gastrointestinal Diseases, ${ }^{\dagger}$ Biodonostia Health Research Institute-Donostia University Hospital, Ikerbasque, CIBERehd, University of the Basque Country (UPV/EHU), San Sebastian, Spain; the Division of Surgery, Inflammatory Medicine and Transplantation, ${ }^{\ddagger}$ Norwegian PSC Research Center, and the Division of Surgery, Inflammatory Medicine and Transplantation, ${ }^{\Uparrow}$ Research Institute of Internal Medicine, Oslo University Hospital, Rikshospitalet, Oslo, Norway; and the K.G. Jebsen Inflammation Research Centre,$^{\S}$ Institute of Clinical Medicine, University of Oslo, Oslo, Norway

\author{
Accepted for publication \\ October 11, 2016. \\ Address correspondence to \\ Luca Maroni, M.D., Ph.D. \\ Department of Gastroenter- \\ ology, Università Politecnica \\ delle Marche, Nuovo Polo \\ Didattico, III Piano, Via \\ Tronto 10, 60020 Ancona, \\ Italy. E-mail: luca.maroni@ \\ live.it.
}

\begin{abstract}
Microbial products are thought to influence the progression of cholangiopathies, in particular primary sclerosing cholangitis (PSC). Inflammasomes are molecular platforms that respond to microbial products through the synthesis of proinflammatory cytokines. We investigated the role of inflammasome activation in cholangiocyte response to injury. Nucleotide-binding oligomerization domain (NOD)-like receptor family, pyrin domain-containing protein 3 (Nlrp3) expression was tested in cholangiocytes of normal and cholestatic livers. Effects of Nlrp3 activation induced by incubation with lipopolysaccharide and ATP was studied in vitro in normal and siRNA-Nlrp3 knocked-down cholangiocytes. Wild-type and Nlrp3 knockout $\left(\mathrm{Nlrp3}^{-/-}\right)$mice were fed 3,5-diethoxycarbonyl-1,4-dihydrocollidine (DDC; a model of sclerosing cholangitis) for 4 weeks. Nlrp3 and its components were overexpressed in cholangiocytes of mice subjected to DDC and in patients affected by PSC. In vitro, Nlrp3 activation stimulated expression of Il-18 but not of Il$1 \beta$ and Il-6. Nlrp3 activation had no effect on cholangiocyte proliferation but significantly decreased the expression of Zonulin-1 and E-cadherin, whereas Nlrp3 knockdown increased the permeability of cholangiocyte monolayers. In vivo, the DDC-stimulated number of cytokeratin-19-positive cells in the liver of wild-type animals was slightly reduced in $\mathrm{N} / \mathrm{rp} 3^{-/-}$mice, and expression of E-cadherin was reestablished. In conclusion, Nlrp3 is expressed in reactive cholangiocytes, in both murine models and patients with PSC. Activation of Nlrp3 leads to synthesis of proinflammatory cytokines and influences epithelial integrity of cholangiocytes. (Am J Pathol 2017, 187: 366-376; http://dx.doi.org/10.1016/j.ajpath.2016.10.010)
\end{abstract}

Cholangiopathies are a broad spectrum of diseases that selectively target cholangiocytes, the epithelial cells lining the biliary tree. In response to injury, cholangiocytes undergo extensive modifications of their phenotype, become reactive, and play essential roles in the regulation of biliary inflammation and development of liver fibrogenesis. ${ }^{1,2}$

Considerable amounts of bacterial products known as pathogen-associated molecular patterns (PAMPs) originating from the gut are directed to the liver by portal circulation, particularly during the course of liver diseases. ${ }^{3}$ Hepatocytes and Kupffer cells are the main cell types responsible for the metabolism and clearance of bacterial products, but bioactive forms of bacterial products such as lipopolysaccharide (LPS) are present in bile and interact with cholangiocytes. ${ }^{4-6}$ Not

Supported by Ministry of Education, dell'Università e della Ricerca grant PRIN 2009 - prot. 2009X84L84_003 (M.M.), Ministry of Health grant GR-2010-2306996 (M.M.), Spanish Ministries of Economy and Competitiveness grant PI15/01132 (J.M.B.) cofinanced by Fondo Europeo de Desarrollo Regional (FEDER), Spanish Carlos III Health Institute (ISCIII) Ciberehd and Miguel Servet Program grant CON14/00129 (J.M.B.), and the Norwegian PSC research center (T.H.K. and E.M.).

Disclosures: None declared. 
surprisingly, biliary cells display a wide spectrum of immunerelated pathways and receptors, such as NF- $\mathrm{KB}$ and Toll-like receptors and react to immune stimuli. ${ }^{7}$ The interactions between cholangiocytes and bacterial products originating from the intestine seem particularly relevant to the pathogenesis of primary sclerosing cholangitis (PSC), in light of its strong association with inflammatory bowel disease. ${ }^{8}$ In the course of PSC, cholangiocytes have been shown to accumulate abnormal levels of LPS and to display an increased response and loss of tolerance to LPS stimulation. ${ }^{6,9}$ However, the pathophysiological relevance of these mechanisms in the development and progression of cholangiopathies remains to be elucidated.

Nucleotide-binding oligomerization domain (NOD)-like receptor (NLR) family, pyrin domain-containing protein 3 (NLRP3) is a recently described member of the innate immune system, belonging to the NLRs. NLRP3 is formed by a molecular complex, known as NLRP3 inflammasome, which is activated by both PAMPs and endogenous danger signals. ${ }^{10}$ As a result, NLRP3 interacts with other members of the inflammasome complex with the subsequent activation of caspase- 1 and the final secretion of proinflammatory cytokines IL-1 $\beta$ and IL- $18 .^{11}$

In the present study we evaluated the role of inflammasome activation in the biological response to injury of cholangiocyte. Thus, we aimed to answer the following questions: i) is Nlrp3 inflammasome expressed in proliferating cholangiocytes? ii) does Nlrp3 activation lead to synthesis of proinflammatory cytokines in cholangiocytes? iii) does Nlrp3 activation affect cholangiocyte biological response to injury? and iv) does Nlrp3 affect the development and progression of liver injury in the experimental model of sclerosing cholangitis?

\section{Materials and Methods}

\section{Materials}

3,5-Diethoxycarbonyl-1,4-dihydrocollidine (DDC) was purchased from ssniff Spezialdiäten $\mathrm{GmbH}$ (Soest, Germany). Anti-cytokeratin-19 (CK-19) (TROMA-III) antibodies were obtained from the Developmental Studies Hybridoma Bank developed under the auspices of the National Institute of Child Health and Human Development and maintained by the University of Iowa, Department of Biological Sciences, Iowa City, IA. Cultured normal rat cholangiocytes (NRCs) were a generous gift of Prof. Gianfranco Alpini (Texas A\&M University, Temple, TX). $\mathrm{Nlrp3}^{\mathrm{A} 350 \mathrm{VneoR}}\left(\mathrm{Nlrp}^{-/-}\right)$mice were purchased from The Jackson Laboratory (Bar Harbor, ME).

\section{Expression of Nlrp3 Inflammasome Components in Cholangiocytes}

The expression levels of the inflammasome components were evaluated by real-time PCR in cholangiocytes isolated from both normal mice and mice fed $0.1 \%$ DDC for 8 weeks, as a model of sclerosing cholangitis. ${ }^{12}$ Purification of cholangiocytes from mice was performed by immune-bead purification, as previously reported. ${ }^{13}$ NLRP3 expression was evaluated by immunohistochemistry in liver sections of patients affected by a typical form of PSC $(n=7)$ or in control subjects $(n=3)$ with non-alcoholic steatohepatitis or non-cholestatic liver disease with nonsignificant ductular reaction. All samples were collected at time of surgical liver resection or liver transplantation. Clinical data and laboratory findings of enrolled patients are summarized in Supplemental Table S1.

\section{Effect of Nlrp3 Inflammasome Activation in Cholangiocytes in Vitro}

Primary culture of NRCs were used for all in vitro experiments. ${ }^{14}$ To test the specificity of inflammasome activation, NRCs were exposed for 48 hours to specific siRNAs against Nlrp3 or to corresponding nontargeting RNAs as described below. Nlrp3 inflammasome activation was induced in cholangiocyte cultures by incubation with LPS $(200 \mathrm{ng} / \mathrm{mL}$ for 24 hours $)$, followed by administration of ATP $(5 \mathrm{mmol} / \mathrm{L}$ for 1,3 , and 6 hours), as previously reported. ${ }^{15}$ Changes in mRNA expression levels of Nlrp3, Il-18, Il-6, Il-1 $\beta, \beta$-catenin, S100A4, and Vimentin were assessed by real-time PCR. Changes in cell proliferation were measured by assessing mRNA levels of Pcna and Ki-67 in cultured cholangiocytes. Epithelial integrity was studied by Western blot analysis for Zonulin-1 and E-cadherin, both markers of epithelial cells and involved in epithelial barrier functions. ${ }^{16,17}$ Epithelial permeability was studied using a commercially available permeability assay kit (catalog no. ECM644; Millipore, Darmstadt, Germany), according to the instructions of the vendor. Briefly, NRC cells were plated on cell culture inserts at a concentration of $5 \times 10^{5}$ cells/well and cultured with a pool of nontarget or Nlrp3 siRNAs for 2 days until a monolayer was formed. The cell culture inserts were transferred to a fresh 24-well plate containing only Dulbecco's modified Eagle's medium: nutrient mixture F-12 (DMEM/F12). The culture medium was replaced with fluorescein isothiocyanate-labeled dextran diluted in Dulbecco's modified Eagle's medium and incubated in the dark for 20 minutes at room temperature. The amount of fluorescein isothiocyanate-labeled dextran diffused through the monolayer into the receiver wells was measured using a microplate reader (Tecan, Mannedorf, Switzerland), with 485-nm excitation wavelength and $535-\mathrm{nm}$ emission wavelength.

\section{Real-Time PCR}

Total RNA was extracted using TRIzol reagent (Life Technologies Corporation, Woburn, MA) according to the manufacturer's protocol. Total RNA $(1 \mu \mathrm{g})$ was converted to cDNA with random primers using the High Capacity cDNA Reverse Transcription Kit (Applied Biosystems, Foster City, CA). Primers for real-time PCR were designed 
with Oligo software version 6.71 (Molecular Biology Insight, Cascade, CO) using reference mRNA sequences accessed through GenBank; the specificity of primers was confirmed by BLAST (Basic Local Alignment Search Tool) analysis. Primer sequences are listed in Table 1. Real-time PCR was performed using the Rotor-Gene 6000 (Corbett Life Science Pty. Ltd., Mortlake, NSW, Australia) with SYBR Green fluorophore. Peptidylprolyl isomerase B (Cyclophilin B) mRNA was used as a reference gene for normalization. The Gene Expression Macro Genex developed by Bio-Rad (Milan, Italy) was used to calculate relative expression values from real-time PCR data.

\section{Immunoblot Analysis}

A total of $50 \mu \mathrm{g}$ of total proteins from liver lysates or cell culture was used in the experiments. After protein electrophoresis and transfer, membranes were incubated overnight at $4^{\circ} \mathrm{C}$ with the specific antibody, followed by incubation with the corresponding secondary antibody. Proteins were visualized using chemiluminescence (ECL Plus Western Blotting Substrate; Pierce, Waltham, MA). The intensity of the bands was determined by scanning video densitometry using the Chemi Doc imaging system (UVP, LCC, Upland, CA).

\section{Transient siRNA Transfection}

The siRNAs were purchased from Dharmacon (Epsom, United Kingdom). The four sense sequences, targeted against Nlrp3 mRNA and identified from GenBank (http://www.ncbi.nlm. nih.gov; accession number NM_001191642.1), were 5'GCAACAACGACCUGGGCGA-3', 5'-AAUUACGGCAAGUUCGAAA- $3^{\prime}, 5^{\prime}$-AGAUUACCCACCCGAGAAA- $3^{\prime}$, and

Table 1 List of Primer Sequences for Real-Time PCR Experiments

\begin{tabular}{ll}
\hline Gene & Sequence \\
\hline IL1B forward & $5^{\prime}$-CAGGAAGGCAGTGTCACTCA-3' \\
IL1B reverse & $5^{\prime}$-AAAGAAGGTGCTTGGGTCCT-3' \\
IL6 forward & $5^{\prime}$-CCGGAGAGAGACTTCACAG-3' \\
IL6 reverse & $5^{\prime}$-CAGAATTGCCATTGCACAAC-3' \\
IL18 forward & $5^{\prime}$-ACCGCAGTAATACGGAGCAT-3' \\
IL18 reverse & $5^{\prime}$-CAGTCTGGTCTGGGATTCGT-3' \\
NLrp3 forward & $5^{\prime}$-AAGTAAGGCCGGAATTCACC-3' \\
NLrp3 reverse & $5^{\prime}$-AAAATGCCTTGGGAGACTCA-3' \\
Ki67 forward & $5^{\prime}$-CCTTGCAGCATTACAAAGCA-3' \\
Ki67 reverse & $5^{\prime}$-GCTTCTCACCTGTTGCTTCC-3' \\
PCNA forward & $5^{\prime}$-TTGGAATCCCAGAACAGGAG-3' \\
PCNA reverse & $5^{\prime}$-AGAAAACTTCACCCCGTCCT-3' \\
Vimentin forward & $5^{\prime}$-CCCTCACCTGTGAAGTGGAT-3' \\
Vimentin reverse & $5^{\prime}$-TCCAGCAGCTTCCTGTAGGT-3' \\
E-cadherin forward & $5^{\prime}$-GACAACGCTCCCATCCCA-3' \\
E-cadherin reverse & $5^{\prime}$-CCACCTCCTTCTTCATCATAG-3' \\
S100A4 forward & $5^{\prime}$-TTGAGCTCTGTCTTGTTCAGC-3' \\
S100A4 reverse & $5^{\prime}$-CTCTATTCAGCACTTCCTCTCTC-3' \\
B-Catenin forward & $5^{\prime}$-ACACAACCTTTCCCACCATCGAGA-3' \\
B-Catenin reverse & $5^{\prime}$-TAGCAGGAGATTATGCAGCGTGGT-3' \\
\hline
\end{tabular}

5'-GGAGGUGGACUGCGAGAGA- ${ }^{\prime}$. NRCs were transfected the day after plating at $30 \%$ to $40 \%$ confluence by using the INTERFERin reagent (PolyPlus Transfection, Illkirch, France), according to the manufacturer's recommendation. siRNA ( $10 \mathrm{nmol} / \mathrm{L})$ was transfected in complete medium. After 48 hours, cells were stimulated with LPS + ATP as previously described $^{15}$ or lysed for RNA isolation. Nlrp3, Il-6, and II-18 transcript levels were analyzed by real-time PCR in NRCs transfected with either the nontargeting siRNA or the Nlrp3 siRNA.

\section{Effect of Nlrp3 Deficiency on Cholangiocyte Response to Injury in Vivo}

Adult (8-week-old) wild-type (WT) or Nlrp3 knockout $\left(N \operatorname{Nlp} 3^{-/-}\right)$mice $(n=5$ per each experimental group) were subjected to $0.1 \%$ DDC feeding for 4 weeks, as a model of sclerosing cholangitis as previously reported. ${ }^{12}$ Changes in cholangiocyte proliferation were determined by measuring differences in intrahepatic small and large bile duct mass (by quantitative immunohistochemistry for CK-19 in liver sections), as previously reported by our group ${ }^{18}$ and by semiquantification of Proliferating Cell Nuclear Antigen (PCNA) immunohistochemistry. For the determination of bile duct mass by computerized analysis, we calculated the volume percentage of the liver occupied by ducts from the total number of points over hepatic tissue and the number of points over CK- $19^{+}$ducts. ${ }^{13}$ Changes in collagen deposition were assessed by quantitative histochemistry for Sirius Red. Semiquantitative assessment of epithelial integrity was made by immunostaining for E-cadherin on formalin-fixed, paraffin-embedded liver sections. Changes in the expression levels of E-cadherin, S100A4, and Vimentin were evaluated by real-time PCR in whole-liver lysate. Animal study protocols were performed in compliance with local institution guidelines.

\section{Statistical Analysis}

Data are expressed as means $\pm \mathrm{SD}$, and $95 \%$ CIs were calculated. Differences between groups were analyzed by analysis of variance. Differences between groups were considered significant when the $P$ value was $<0.05$.

\section{Results}

\section{Nlrp3 Is Expressed in Reactive Cholangiocytes}

The expression of the Nlrp3 inflammasome was increased in both experimental and human conditions of sclerosing cholangitis. Cholangiocytes isolated from WT mice subjected to 8 weeks of DDC treatment showed elevated Nlrp3 mRNA levels compared with cholangiocytes of mice administered a control diet. In line with this, the mRNA expression of the adaptor protein Asc was found increased in reactive cholangiocytes (Figure 1A). 
Moreover, NLRP3 expression was semiquantitatively assessed by immunohistochemistry in liver sections of control individuals and patients affected by the typical form of PSC. As shown in Figure 1B, no signal could be detected in the biliary cells of the normal human liver. However, NLRP3 immunostaining showed positive expression in reactive cholangiocytes in livers of PSC patients, thus highlighting that activation of the NLRP3 inflammasome might play a role in this human disease. Clinical and laboratory findings of the PSC patients investigated in the study are reported in Supplemental Table S1.

\section{Nlrp3 Activation Promotes the Expression of Il-18 but Not Il-1 $\beta$ and Il-6}

Nlrp3 inflammasome activation was induced in vitro by sequential incubation with LPS and ATP (LPS+ATP), as previously reported. ${ }^{15}$ LPS +ATP stimulation of cultured cholangiocytes resulted in increased mRNA expression of Nlrp3 (Figure 2A). Incubations with LPS alone (or ATP alone; data not shown) did not induce any effect on Nlrp3 mRNA synthesis. Stimulation with LPS+ATP induced the expression of the proinflammatory cytokines Il-18 and Il-6, with the most prominent effect after 3 hours and 1 hour of ATP incubation, respectively. Nlrp3 activation had no effect on the synthesis of Il-1 $\beta$ in cultured cholangiocyte (Figure 2, B-D).

To verify that the effects of LPS+ATP stimulation were directly Nlrp3 dependent, Nlrp3 was knocked down in cultured cholangiocytes by a specific siRNA. We achieved a basal knockdown level of approximately $80 \%$ in Nlrp3 expression, which was paralleled by a fourfold decrease in Nlrp3 expression on stimulation with
LPS +ATP (Figure 3A). Moreover, Nlrp3 knockdown abolished the increase in Il-18 synthesis in cells incubated with LPS+ATP (Figure 3B), whereas it had no effect on the increased expression of Il-6, indicating an inflammasome-independent effect in the latter (Figure 3C).

\section{Nlrp3 Activation Has No Effect on Cholangiocyte Proliferation but Influences Epithelial Barrier Function in Vitro}

To verify whether Nlrp3 activation influences biliary proliferation, cultured cholangiocytes were exposed to LPS +ATP. As shown in Figure 4, the incubation with LPS+ATP did not determine any significant effect on cultured cholangiocyte proliferation with either of the assays used. However, the activation of the Nlrp3 inflammasome reduced the protein expression of E-cadherin and Zonulin-1, both markers of epithelial cells and involved in epithelial barrier functions, compared with control cells (Figure 5A). To confirm that the effects of LPS+ATP incubation were mediated by Nlrp3, the experiments were repeated in the absence or presence of a specific siRNA for Nlrp3. As shown in Figure 5B, the knockdown of Nlrp3 neutralized the decreases in Zonulin-1 and E-cadherin expression induced by LPS +ATP, thus indicating the involvement of Nlrp3 activation in epithelial integrity in vitro. On a functional level, permeability of fluorescein isothiocyanate-dextran in cells stimulated by LPS +ATP was further increased in Nlrp3 knocked-down cholangiocytes compared with control cells (Figure 5C). LPS +ATP incubation also induced the expression of $\beta$-catenin, which was blunted in Nlrp3 knocked-down cholangiocytes (Supplemental Figure S1).
A
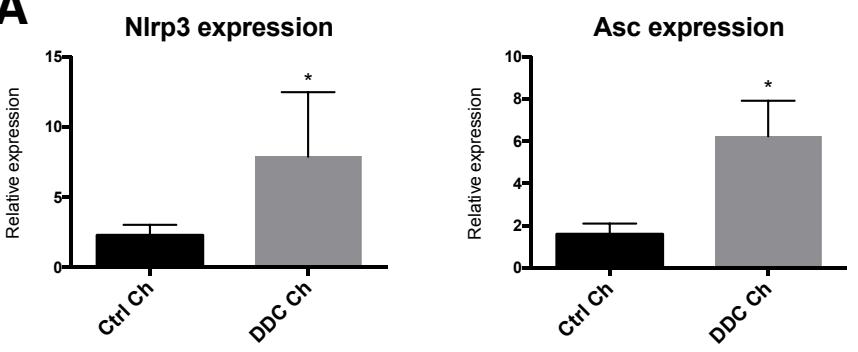

B

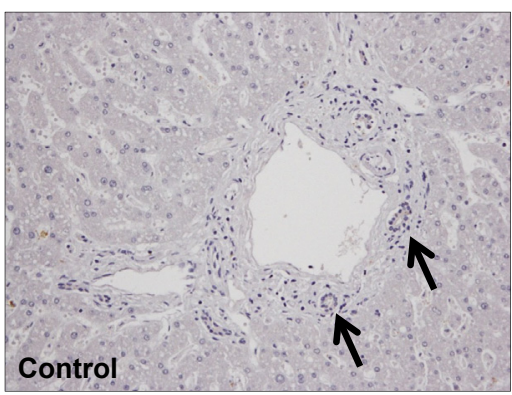

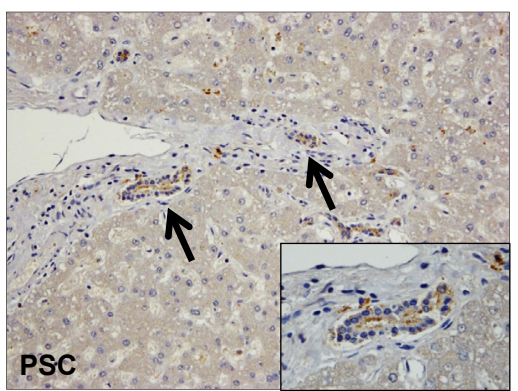

Figure 1 Expression of Nlrp3 in reactive cholangiocytes. A: Cholangiocytes were isolated from WT mice subjected to 8 weeks of control or DDCcontaining diet, as a model of sclerosing cholangitis. mRNA expression levels of Ntrp3 and of the adaptor protein Asc resulted in increased DDC cholangiocytes compared with controls. B: Normal human liver (left panel) was negative for NLRP3 immunohistochemistry. Immunohistochemistry showed a positive staining for NLRP3 in cholangiocytes of liver sections from patients affected by PSC (right panel). Arrows indicate bile ducts. Inset shows Magnification of $\mathrm{NLRP3}^{+}$bile duct of PSC patient. Data are expressed as means \pm SD. $n=3$ or more experiments (A). ${ }^{*} P<0.05$ versus control cholangiocytes. Original magnification: $\times 20$ (B, main image); $\times 40$ (inset). Ch, cholangiocyte; Ctrl, control; DDC, 3,5-diethoxycarbonyl-1,4-dihydrocollidine; Nlrp3, nucleotide-binding oligomerization domain (NOD)like receptor (NLR) family, pyrin domain-containing protein 3; PSC, primary sclerosing cholangitis; WT, wild-type. 
A

NIrp3 expression

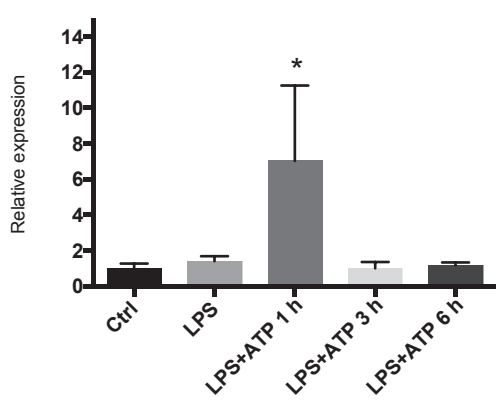

C

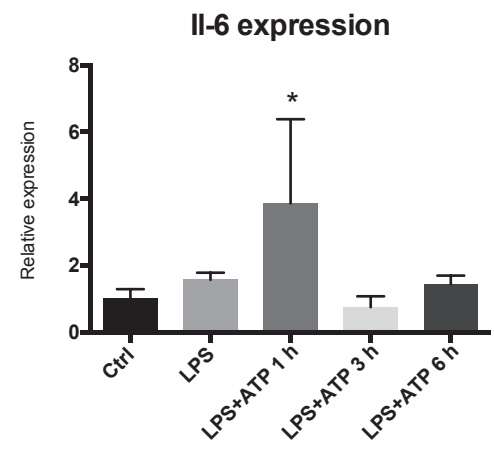

B

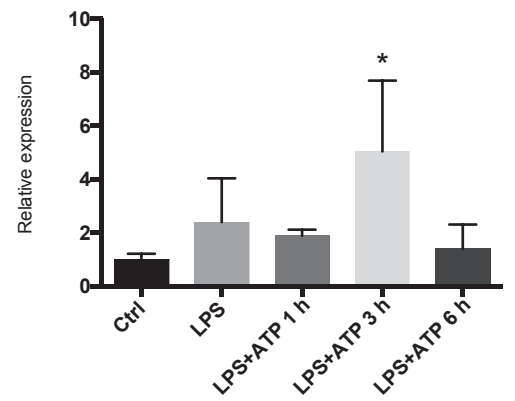

D

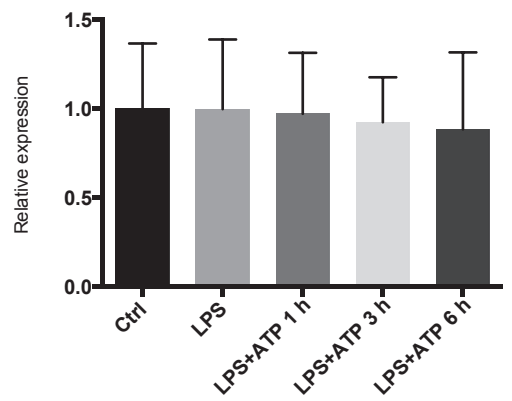

Figure 2 Effect of Nlrp3 activation in cultured cholangiocytes. Nlrp3 inflammasome activation was induced in cultured cholangiocytes by sequential incubation with LPS $(200 \mathrm{ng} / \mathrm{mL}$, for 24 hours) followed by administration of ATP ( $5 \mathrm{mmol} /$ $\mathrm{L}$, for 1,3 , and 6 hours). A: Incubations with LPS+ATP induced the mRNA expression of Nlrp3 in cultured cholangiocytes. B and C: The same incubations determined a significant increase in the mRNA expression levels of Il-18 (B) and Il-6 (C). D: LPS+ATP stimulation had no effect on Il-1 $\beta$ synthesis. Data are expressed as means \pm SD. $n=3$ or more experiments. ${ }^{*} P<0.05$ versus control (analysis of variance). Ctrl, control; LPS, lipopolysaccharide; Nlrp3, nucleotide-binding oligomerization domain (NOD)-like receptor (NLR) family, pyrin domain-containing protein 3 .

\section{Nlrp3 Regulates Cholangiocyte Proliferation in a Mouse Model of Sclerosing Cholangitis}

To study the influence of Nlrp3 activation in an in vivo model of sclerosing cholangitis, WT and $\mathrm{Nlrp}^{-1-}$ mice were fed with a DDC-containing diet for 4 weeks. The increase in bile duct mass induced by 4 weeks of DDC feeding was slightly, but significantly, reduced in $N l r p 3^{-/-}$ mice, as assessed by quantitative CK-19 immunohistochemistry, and compared with control mice (Figure 6A). To support the in vitro data showing a decreased expression of cell adhesion molecules, E-cadherin expression was semiquantitatively assessed by immunohistochemistry. As shown in the representative images of Figure 6B, expression of E-cadherin was found increased in $\mathrm{Nlrp3}^{-1-}$ mice subjected to DDC compared with control mice. mRNA expression level of E-cadherin was also significantly increased in $\mathrm{Nlrp} 3^{-/-}$mice, whereas there were no differences in the expression of S100A4 and Vimentin (Supplemental Figure S2). Quantitative histochemistry for
A

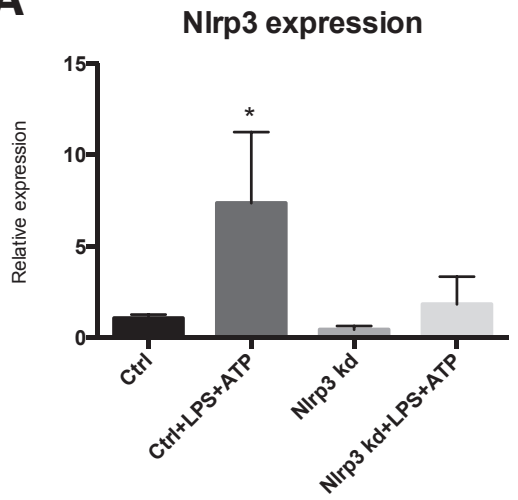

B

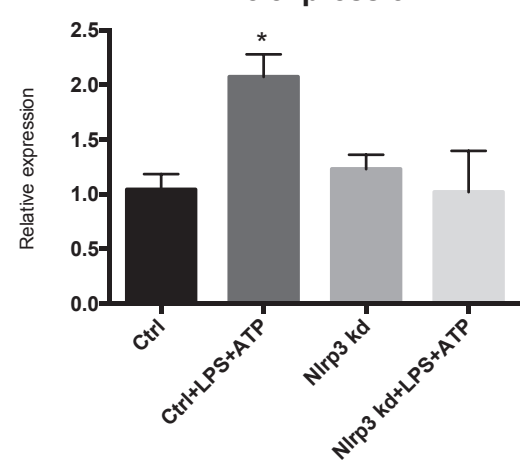

C

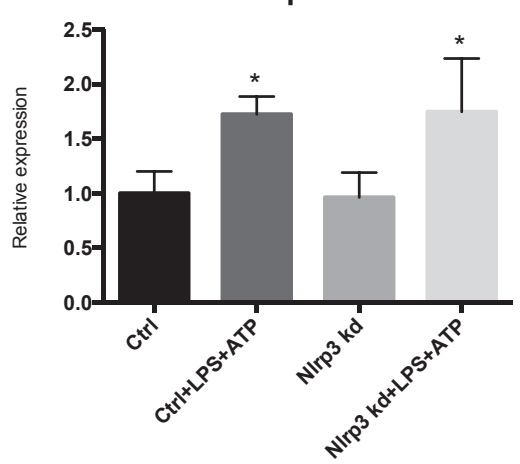

Figure 3 Effect of Nlrp3 knockdown on Nlrp3, Il-18, and Il-6 synthesis. Nlrp3 expression was knocked down in cultured cholangiocytes by specific siRNAs, and cells were subsequently stimulated by LPS+ATP. A: Nlrp3 knockdown significantly reduced Nlrp3 mRNA expression in cells stimulated with LPS+ATP. B: Moreover, the induction of Il-18 expression stimulated by LPS+ATP was abolished by Nlrp3 knockdown. C: Nlrp3 knockdown had no effect on the synthesis of Il-6 stimulated by LPS+ATP incubation, suggesting an indirect inflammasome-independent effect of the incubation. Data are expressed as means \pm SD. $n=3$ or more experiments. ${ }^{*} P<0.05$ versus control. Ctrl, control; kd, knocked down; LPS, lipopolysaccharide; Nlrp3, nucleotide-binding oligomerization domain (NOD)-like receptor (NLR) family, pyrin domain-containing protein 3. 
A

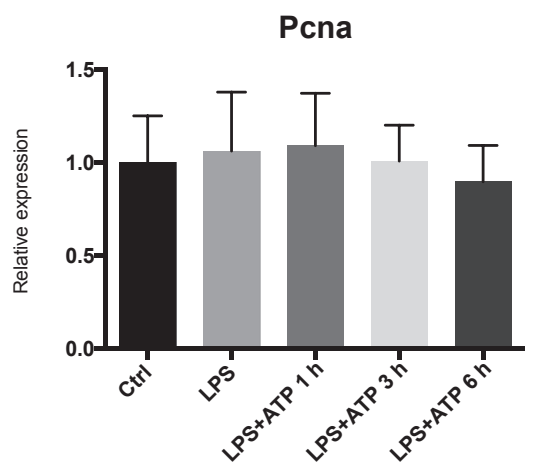

B

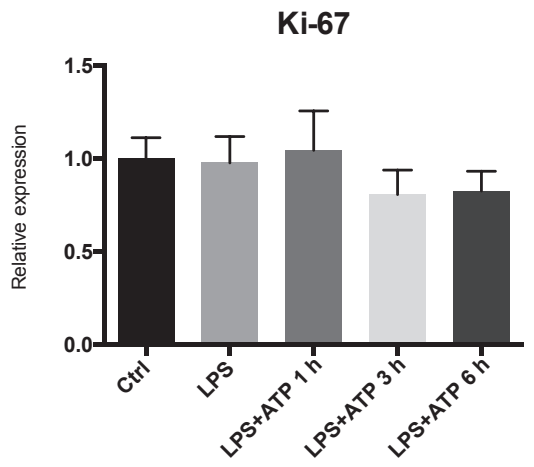

Figure 4 Effect of Nlrp3 activation on cholangiocyte proliferation in vitro. Nlrp3 inflammasome activation was induced by LPS+ATP incubations, and cholangiocyte proliferation was assessed by qPCR for Pcna (A) and Ki-67 (B). Nlrp3 activation had no effect on the expression of both Pcna and Ki-67, indicating that the inflammasome activation has no effect of cholangiocyte proliferation in vitro. Data are expressed as means \pm SD. $n=3$ or more experiments. Ctrl, control; LPS, lipopolysaccharide; Nlrp3, nucleotide-binding oligomerization domain (NOD)-like receptor (NLR) family, pyrin domain-containing protein 3; qPCR, real-time quantitative PCR.
Sirius Red revealed a trend toward a reduced collagen deposition in the liver of $\mathrm{Nlrp}^{-1-}$ mice compared with WT animals, but the difference was not statistically significant (Supplemental Figure S3).

\section{Discussion}

In the present study, we show that i) the expression of the Nlrp3 inflammasome is increased in reactive cholangiocytes, both in a murine model of sclerosing cholangitis and in human PSC; ii) the activation of the Nlrp3 inflammasome in cholangiocyte leads to secretion of Il-18; iii) in vitro, Nlrp3 activation influences the epithelial barrier function of cholangiocytes but has no effects on biliary proliferation; and iv) in vivo, the lack of Nlrp3 has a minimal effect on cholangiocyte proliferation in response to injury.

Despite being sterile in normal conditions, the biliary tract is constantly exposed to microorganisms and to a wide variety of bacterial products known as PAMPs. Bacteria can colonize the bile ducts due to the continuity with the duodenum, which occasionally results in infection of the biliary tree and cholangitis. ${ }^{19,20}$ Moreover, the portal circulation delivers to the hepatocytes considerable amounts of gutderived PAMPs such as LPS, which eventually reach the bile in a bioactive form. ${ }^{4,21}$

The role of the activation of cholangiocyte immune pathways mediated by PAMPs in the pathogenesis of cholangiopathies such as PSC remains to be fully evaluated. Interestingly, cholangiocytes of PSC patients have been shown to accumulate abnormal amounts of LPS compared with other cholestatic conditions. ${ }^{6}$ Multiple studies have shown that LPS stimulation of cholangiocytes activates a variety of molecular pathway that are involved in cytokines synthesis, biliary proliferation, and cell survival. ${ }^{22-24}$ The effects of innate immunity activation in cholangiocytes are particularly interesting in the setting of PSC, given its strong association with inflammatory bowel diseases. An increased intestinal permeability or a particular genetic susceptibility to bacterial products in the PSC-inflammatory bowel disease form is the basis of the so-called leaky gut hypothesis to explain the pathogenesis of PSC. ${ }^{25}$ Of note, combined treatment with ursodeoxycholic acid and the antibiotic metronidazole has proven to be more effective than ursodeoxycholic acid alone in reducing alkaline phosphatase levels and the New Mayo Risk Score in PSC patients. ${ }^{26}$

Together with a wide variety of pathogen pattern recognition receptors such as Toll-like receptors, ${ }^{7}$ we showed that reactive cholangiocytes express the recently described NLR family, pyrin domain-containing protein 3 (Nlrp3) inflammasome (Figure 1). The NLRP3 is a cytosolic sensor that is activated by a number of insults originating from both exogenous and endogenous stimuli. ${ }^{27}$ On activation, NLRP3 units oligomerize and form the inflammasome complex by interacting with apoptosis-associated speck-like protein containing a CARD [C-terminal caspase recruitment domain] (ASC), which in turn binds to and activates caspase-1. ${ }^{10,28}$ The final result of NLRP3 activation is the secretion of proinflammatory cytokines such as IL- $1 \beta$ and IL-18, which have been found in a variety of tissues, including the liver. ${ }^{29,30}$ As shown in Figure 1A, Nlrp3 and inflammasome components such as Asc were up-regulated in reactive cholangiocytes isolated from mice subjected to DDC treatment, as a model of sclerosing cholangitis. Interestingly, although NLRP3 protein expression was not detected in biliary cells of control subjects, a positive immunostaining for NLRP3 is present in PSC cholangiocytes (Figure 1B), indicating the up-regulation of this pathway also in the human disease.

To test that the activation of the Nlrp3 inflammasome has a functional role in biliary cells, we stimulated cultured cholangiocytes with LPS+ATP, as previously described. ${ }^{15}$ This experimental approach aimed at verifying the effect of inflammasome activation in cholangiocytes, and we cannot rule out the possible contribution of different PAMPs in this setting. LPS +ATP resulted in increased expression of Nlrp3 and of the proinflammatory cytokines Il-18 and Il-6 (Figure 2, A-C). However, we could not identify any effect of Nlrp3 activation on Il-1 $\beta$ synthesis in cholangiocytes 
A

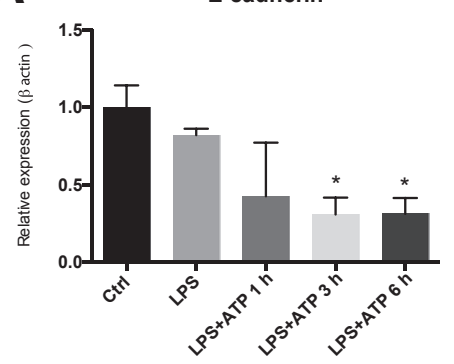

B

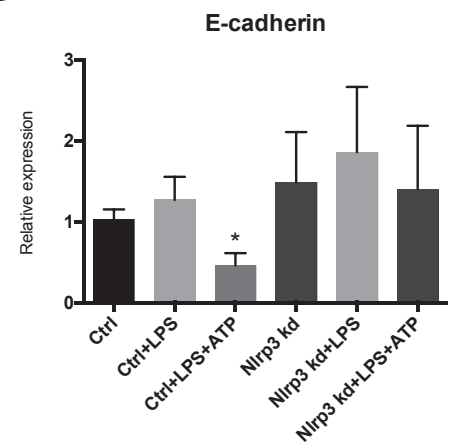

C

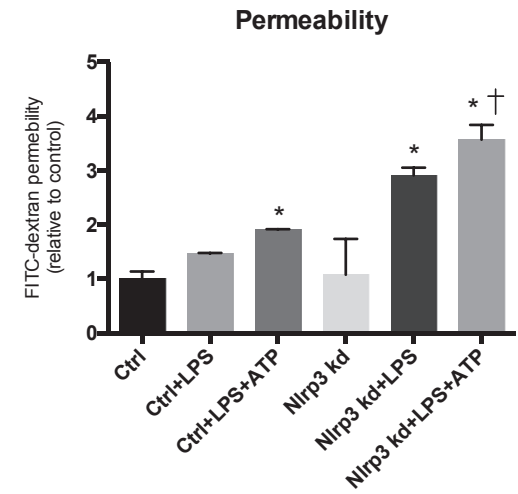

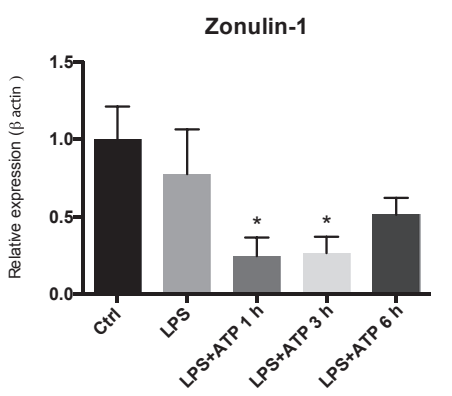

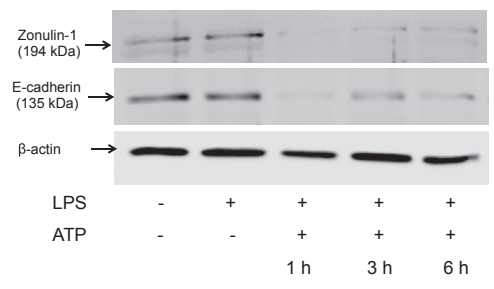

Zonulin-1
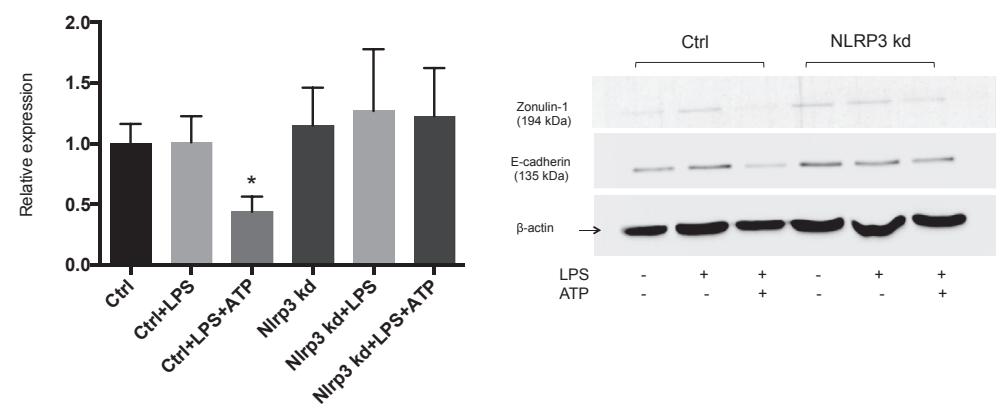

Figure 5 Effect of Nlrp3 activation on epithelial integrity in vitro. A: The activation of the Nlrp3 inflammasome by incubation with LPS+ATP induced a significant decrease in the protein expression of E-cadherin (left) and Zonulin1 (middle), both markers of epithelial integrity. Right panel shows representative Western blot analysis. B: The knockdown of Nlrp3 reestablished the expression of both E-cadherin (left) and Zonulin-1 (middle) in cholangiocytes stimulated by LPS+ATP. Right panel shows representative Western blot analysis. C: LPS+ATP stimulated the permeability of control cells measured by the permeation of FITC-dextran through an epithelial monolayer; this effect was significantly increased in Nlrp3 knocked-down cholangiocytes. Data are expressed as means \pm SD. $n=3$ or more experiments. ${ }^{*} P<0.05$ versus control; ${ }^{\dagger} P<0.05$ versus control+LPS+ATP. Ctrl, control; FITC, fluorescein isothiocyanate; kd, knocked down; LPS, lipopolysaccharide; Nlrp3, nucleotidebinding oligomerization domain (NOD)-like receptor (NLR) family, pyrin domain-containing protein 3.

(Figure 2D). When Nlrp3 expression was knocked down by a specific siRNA, the increases in Nlrp3 and Il-18 were abolished (Figure 3); in contrast, Nlrp3 knockdown had no effect on the expression of Il-6, indicating that the latter was an inflammasome-independent effect of the incubation.

Cholangiocytes lining the bile duct are quiescent cells in normal conditions. In response to damage, however, biliary cells undergo extensive modification of their biology, start to proliferate, and acquire a neuroendocrine phenotype characterized by the synthesis and the release of neuroendocrine peptides, cytokines, and growth factor. These cholangiocytes have been termed reactive cholangiocytes and are thought to play an essential role in disease progression and development of biliary fibrosis. ${ }^{2,31-33}$ The initial proliferation of cholangiocytes is functional to maintain the biliary mass and, therefore the secretory functions of the biliary epithelium. However, cell death eventually prevails with consequent development of ductopenia, whereas the constant inflammatory stimulation is thought to contribute to cholangiocarcinoma development. ${ }^{34}$ As shown in Figure 4, experimental activation of Nlrp3 inflammasome in cholangiocytes did not have any significant effect on biliary proliferation in vitro, assessed by both Pcna and Ki-67 expression. The direct role of Nlrp3 activation in physiological and neoplastic cell proliferation is yet to be fully elucidated. ${ }^{35}$ In hepatobiliary diseases, despite chronic inflammation being thought to contribute to neoplastic degeneration of regenerative cellular proliferation, a recent report has clearly demonstrated that NLRP3 expression was down-regulated in human hepatocellular carcinoma and correlated with advanced stage of the disease. $^{36}$ 

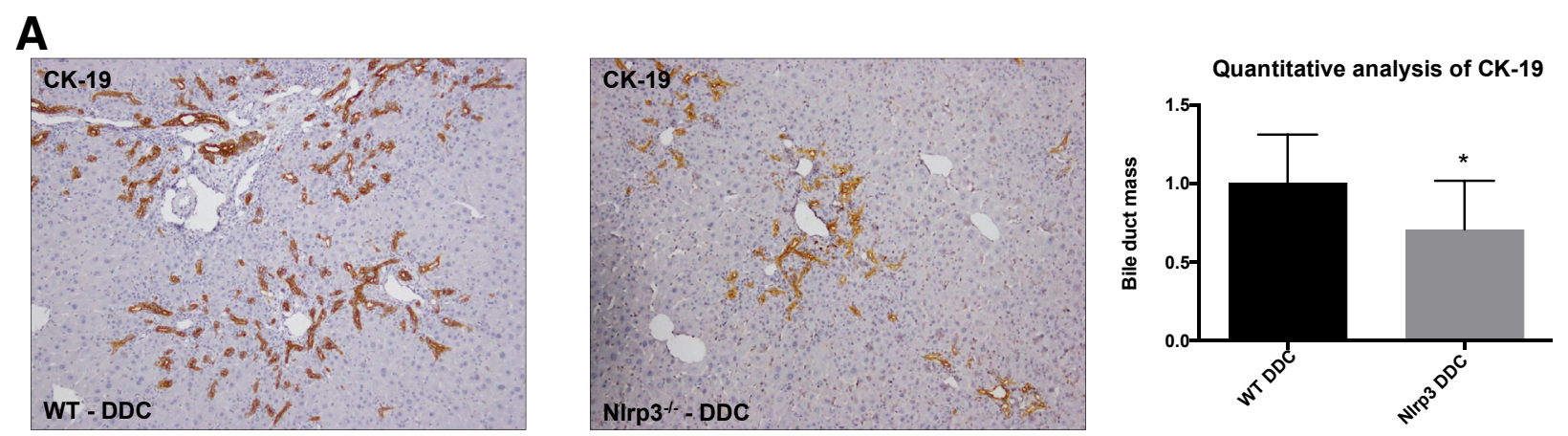

B
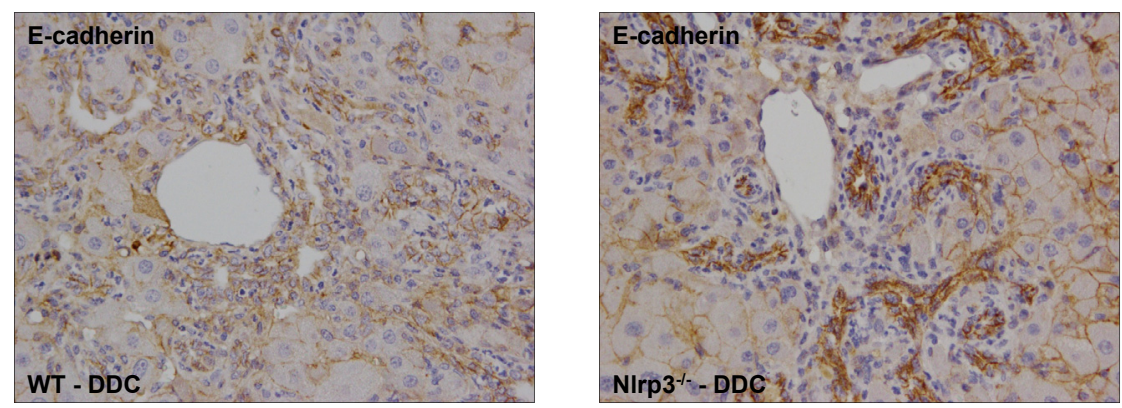

Figure 6 Role of Nlrp3 in cholangiocyte proliferation and epithelial integrity in the 4-week DDC model of sclerosing cholangitis. A: CK-19 immunostaining. WT and $\mathrm{Nlrp}^{-/-}$mice were subjected to 4 weeks of DDC feeding, as a model of sclerosing cholangitis. The increases in bile duct mass (assessed by immunohistochemistry for (K-19) was significantly reduced in DDC-treated NIrp $3^{-/}$mice compared with WT animals. Representative images of CK-19 immunostaining (positive in brown) in WT mice subjected to DDC (left panel) and Nlrp3 ${ }^{-1-}$ mice subjected to DDC feeding (middle panel). Quantification of the immunostaining in WT and Nlrp3 $3^{-/-}$mice (right panel). B: Semiquantitative analysis of E-cadherin immunostaining showed a tendency to increased protein expression in N(rp3 $3^{-/}$mice (right panel) compared with WT mice (left panel) subjected to DDC feeding, suggesting a restored epithelial integrity in $\mathrm{Nlrp3}^{-/-}$mice. Data are expressed as means \pm SEM. ${ }^{*} P<0.05$ versus WT DDC. CK-19, cytokeratin-19; DDC, 3,5-diethoxycarbonyl-1,4-dihydrocollidine; Nlrp3, nucleotide-binding oligomerization domain (NOD)-like receptor (NLR) family, pyrin domain-containing protein 3; WT, wild-type.

The activation of Nlrp3 in cholangiocytes had, however, important effects on cell-adhesion molecules involved in epithelial cell barrier functions. Incubations with LPS +ATP were indeed able to significantly reduce the protein expression of E-cadherin and Zonulin-1 in cultured cholangiocytes (Figure 5A). The specificity of these results was tested in cholangiocytes knocked down for Nlrp3 expression, which completely abolished the effects of LPS +ATP (Figure 5B). On a functional level, the permeability of LPS+ATP-treated cholangiocyte monolayers increased compared with control incubations. Moreover, this effect was more pronounced in Nlrp3 knocked-down cholangiocytes, where E-cadherin and Zonulin-1 protein expression is reestablished (Figure 5C). Decreased protein expression levels of E-cadherin and Zonulin-1 correlate with increased epithelial permeability in different tissues, but an opposite effect has also been described in a number of studies. ${ }^{37-42}$ In particular, the subcellular localization of the proteins, rather than the expression levels, is essential to determine their functional activity. ${ }^{41}$ In our experimental setting, the activation of the Nlrp3 inflammasome seems therefore to blunt the increased epithelial permeability stimulated by LPS +ATP, despite the down-regulation of Ecadherin and Zonulin-1. Our results are in line with previous reports showing that the Nlrp3 is involved in the maintenance of epithelial integrity during experimental colitis. ${ }^{43}$ Nlrp3 activation also induced the expression of $\beta$ catenin, which forms a complex with E-cadherin on the cell membrane (Supplemental Figure S1). Interestingly, an inverse correlation between E-cadherin and $\beta$-catenin expression has been previously reported both in in vitro studies and in immunohistochemical studies in human tissues. $^{44,45}$

The impairment of epithelial barrier function of cholangiocytes plays an essential role in the development of experimental biliary injury and is thought to contribute to the progression of PSC in humans. ${ }^{46}$ Indeed, proinflammatory cytokines and PAMPs may alter cholangiocyte permeability, both with and without structural changes of cell-adhesion molecules. ${ }^{47,48}$ In the $M d r 2^{-/-}$mouse, a murine model of PSC, the disruption of cholangiocyte epithelial integrity has been shown to cause leakage of bile acid from the bile ducts and thereby to determine portal inflammation and fibrosis. ${ }^{49}$ In the present study, we show that the activation of the Nlrp3 inflammasome mediated by PAMPs influences the epithelial barrier function of cholangiocyte in vitro, supporting the concept that microbial products may alter the biology of biliary cells.

To evaluate whether Nlrp3 activation has a significant role in cholangiocyte response to injury in vivo, we fed 
$N l r p 3^{-1-}$ mice with a DDC-containing diet for 4 weeks and compared them with WT mice. As shown in Figure 6A, the increase in bile duct mass stimulated by DDC feeding was evaluated by quantitative immunohistochemistry for CK-19 and was reduced in Nlrp3 $3^{-1-}$ mice compared with controls. The study of cholangiocyte proliferation by immunohistochemistry for PCNA and of the relative expansion of small and large cholangiocytes, evaluated as previously reported by our group ${ }^{18}$ did not change between $N$ lrp $3^{-/-}$mice and WT animals after DDC administration (data not shown). Taken together, these data show that activation of the inflammasome has a minimal role on cholangiocyte proliferation in vivo, thus confirming our in vitro findings. The semiquantitative evaluation of E-cadherin immunohistochemistry showed a tendency to increased expression in bile ducts of DDC-treated $\mathrm{Nlrp}^{-1-}$ mice compared with WT mice (Figure 6B). To support those findings, E-cadherin mRNA expression levels were significantly increased in liver lysates of $\mathrm{Nlrp3}^{-/-}$mice compared with WT mice subjected to DDC treatment (Supplemental Figure S2). Expression of S100A4, which is known to down-regulate Ecadherin expression, and Vimentin, which is inversely correlated with E-cadherin expression in the context of epithelial-mesenchymal transition, ${ }^{50-52}$ was not different between the two groups (Supplemental Figure S2). It has to be underlined that the effects of DDC feeding in our $\mathrm{Nlrp}^{-1-}$ mouse model could be influenced by the lack of the inflammasome in cells other than cholangiocytes. Inflammatory cells, in particular, may have contributed to the final phenotype of $\mathrm{Nlrp3}^{-/-}$mice treated with DDC, given the known expression of the inflammasome in these cells and the pro-proliferative effects of a number of cytokines. ${ }^{53,54}$ We also evaluated the effect of Nlrp3 deficiency on the development of liver fibrosis after DDC administration. Despite collagen deposition showing a reduced trend in $N \mathrm{Nrp}^{-1-}$ mice, the difference was not statistically significant (Supplemental Figure S3). Of note, in our experimental model DDC was administered for 4 weeks mainly to achieve biliary damage and may have determined a lower induction of collagen deposition than regimens of 8 weeks of feeding. ${ }^{12}$

\section{Conclusions}

We showed that the activation of Nlrp3 inflammasome by microbial products induces the synthesis of proinflammatory cytokines and alters the epithelial barrier function of cholangiocytes. Our data suggest that bacterial products may activate the Nlrp3 inflammasome in cholangiocytes and may influence the development of cholangiopathies. Those data show that the Nlrp3 inflammasome affects the biology of reactive cholangiocytes, both in murine models of sclerosing cholangitis and possibly in PSC patients.

Further efforts are needed to clarify the potential role of Nlrp3 inhibition in the course of PSC. In particular, it remains to elucidate the specific nature of Nlrp3 inflammasome activators, especially in the human setting of PSC. Such a study may identify potential molecular targets for therapy and certainly deserves further investigations.

\section{Acknowledgment}

We thank Prof. Gianfranco Alpini for reading the manuscript and making useful suggestions to improve the quality of the article.

\section{Supplemental Data}

Supplemental material for this article can be found at http://dx.doi.org/10.1016/j.ajpath.2016.10.010.

\section{References}

1. Lazaridis KN, Strazzabosco M, Larusso NF: The cholangiopathies: disorders of biliary epithelia. Gastroenterology 2004, 127:1565-1577

2. Alvaro D, Mancino MG, Glaser S, Gaudio E, Marzioni M, Francis H, Alpini G: Proliferating cholangiocytes: a neuroendocrine compartment in the diseased liver. Gastroenterology 2007, 132:415-431

3. Lumsden AB, Henderson JM, Kutner MH: Endotoxin levels measured by a chromogenic assay in portal, hepatic and peripheral venous blood in patients with cirrhosis. Hepatology 1988, 8:232-236

4. Mimura Y, Sakisaka S, Harada M, Sata M, Tanikawa K: Role of hepatocytes in direct clearance of lipopolysaccharide in rats. Gastroenterology 1995, 109:1969-1976

5. Hiramatsu K, Harada K, Tsuneyama K, Sasaki M, Fujita S, Hashimoto T, Kaneko S, Kobayashi K, Nakanuma Y: Amplification and sequence analysis of partial bacterial $16 \mathrm{~S}$ ribosomal RNA gene in gallbladder bile from patients with primary biliary cirrhosis. J Hepatol 2000, 33:9-18

6. Sasatomi K, Noguchi K, Sakisaka S, Sata M, Tanikawa K: Abnormal accumulation of endotoxin in biliary epithelial cells in primary biliary cirrhosis and primary sclerosing cholangitis. J Hepatol 1998, 29: 409-416

7. Chen XM, O'Hara SP, LaRusso NF: The immunobiology of cholangiocytes. Immunol Cell Biol 2008, 86:497-505

8. Lindor KD, Kowdley KV, Harrison ME: ACG Clinical Guideline: primary sclerosing cholangitis. Am J Gastroenterol 2015, 110: 646-659

9. Mueller T, Beutler C, Pico AH, Shibolet O, Pratt DS, Pascher A, Neuhaus P, Wiedenmann B, Berg T, Podolsky DK: Enhanced innate immune responsiveness and intolerance to intestinal endotoxins in human biliary epithelial cells contributes to chronic cholangitis. Liver Int 2011, 31:1574-1588

10. Tschopp J, Schroder K: NLRP3 inflammasome activation: the convergence of multiple signalling pathways on ROS production? Nat Rev Immunol 2010, 10:210-215

11. Davis BK, Wen H, Ting JP: The inflammasome NLRs in immunity, inflammation, and associated diseases. Annu Rev Immunol 2011, 29: 707-735

12. Fickert P, Stoger U, Fuchsbichler A, Moustafa T, Marschall HU, Weiglein AH, Tsybrovskyy O, Jaeschke H, Zatloukal K, Denk H, Trauner M: A new xenobiotic-induced mouse model of sclerosing cholangitis and biliary fibrosis. Am J Pathol 2007, 171:525-536

13. Marzioni M, Saccomanno S, Agostinelli L, Rychlicki C, De Minicis S, Pierantonelli I, Trauner M, Fickert P, Muller T, Shanmukhappa K, Trozzi L, Candelaresi C, Baroni GS, Benedetti A: PDX-1/Hes-1 interactions determine cholangiocyte proliferative 
response to injury in rodents: possible implications for sclerosing cholangitis. J Hepatology 2013, 58:750-756

14. Salter KD, Roman RM, LaRusso NR, Fitz JG, Doctor RB: Modified culture conditions enhance expression of differentiated phenotypic properties of normal rat cholangiocytes. Lab Invest 2000, 80:1775-1778

15. Mao K, Chen S, Chen M, Ma Y, Wang Y, Huang B, He Z, Zeng Y, Hu Y, Sun S, Li J, Wu X, Wang X, Strober W, Chen C, Meng G, Sun B: Nitric oxide suppresses NLRP3 inflammasome activation and protects against LPS-induced septic shock. Cell Res 2013, 23: 201-212

16. Fanning AS, Anderson JM: Zonula occludens-1 and -2 are cytosolic scaffolds that regulate the assembly of cellular junctions. Ann N Y Acad Sci 2009, 1165:113-120

17. Tian X, Liu Z, Niu B, Zhang J, Tan TK, Lee SR, Zhao Y, Harris DC, Zheng G: E-cadherin/beta-catenin complex and the epithelial barrier. J Biomed Biotechnol 2011, 2011:567305

18. Marzioni M, Agostinelli L, Candelaresi C, Saccomanno S, De Minicis S, Maroni L, Mingarelli E, Rychlicki C, Trozzi L, Banales JM, Benedetti A, Baroni GS: Activation of the developmental pathway neurogenin-3/microRNA-7a regulates cholangiocyte proliferation in response to injury. Hepatology 2014, 60:1324-1335

19. Kaya M, Bestas R, Bacalan F, Bacaksiz F, Arslan EG, Kaplan MA: Microbial profile and antibiotic sensitivity pattern in bile cultures from endoscopic retrograde cholangiography patients. World J Gastroenterol 2012, 18:3585-3589

20. Rosch T, Triptrap A, Born P, Ott R, Weigert N, Frimberger E, Allescher HD, Classen M, Kamereck K: Bacteriobilia in percutaneous transhepatic biliary drainage: occurrence over time and clinical sequelae. A prospective observational study. Scand J Gastroenterol 2003, 38:1162-1168

21. Osnes T, Sandstad O, Skar V, Osnes M: Lipopolysaccharides and beta-glucuronidase activity in choledochal bile in relation to choledocholithiasis. Digestion 1997, 58:437-443

22. Yokoyama T, Komori A, Nakamura M, Takii Y, Kamihira T, Shimoda S, Mori T, Fujiwara S, Koyabu M, Taniguchi K, Fujioka H, Migita $\mathrm{K}$, Yatsuhashi $\mathrm{H}$, Ishibashi $\mathrm{H}$ : Human intrahepatic biliary epithelial cells function in innate immunity by producing IL-6 and IL-8 via the TLR4-NF-kappaB and -MAPK signaling pathways. Liver Int 2006, 26:467-476

23. Trussoni CE, Tabibian JH, Splinter PL, O'Hara SP: Lipopolysaccharide (LPS)-induced biliary epithelial cell NRas activation requires epidermal growth factor receptor (EGFR). PLoS One 2015, 10: e0125793

24. O'Hara SP, Splinter PL, Trussoni CE, Gajdos GB, Lineswala PN, LaRusso NF: Cholangiocyte N-Ras protein mediates lipopolysaccharide-induced interleukin 6 secretion and proliferation. J Biol Chem 2011, 286:30352-30360

25. Tabibian JH, Talwalkar JA, Lindor KD: Role of the microbiota and antibiotics in primary sclerosing cholangitis. Biomed Res Int 2013, 2013:389537

26. Farkkila M, Karvonen AL, Nurmi H, Nuutinen H, Taavitsainen M, Pikkarainen P, Karkkainen P: Metronidazole and ursodeoxycholic acid for primary sclerosing cholangitis: a randomized placebocontrolled trial. Hepatology 2004, 40:1379-1386

27. Wen H, Miao EA, Ting JP: Mechanisms of NOD-like receptorassociated inflammasome activation. Immunity 2013, 39:432-441

28. Mariathasan S, Monack DM: Inflammasome adaptors and sensors: intracellular regulators of infection and inflammation. Nat Rev Immunol 2007, 7:31-40

29. Szabo G, Csak T: Inflammasomes in liver diseases. J Hepatology 2012, 57:642-654

30. Wree A, Eguchi A, McGeough MD, Pena CA, Johnson CD, Canbay A, Hoffman HM, Feldstein AE: NLRP3 inflammasome activation results in hepatocyte pyroptosis, liver inflammation, and fibrosis in mice. Hepatology 2014, 59:898-910

31. Park SM: The crucial role of cholangiocytes in cholangiopathies. Gut Liver 2012, 6:295-304
32. Maroni L, Haibo B, Ray D, Zhou T, Wan Y, Meng F, Marzioni M, Alpini G: Functional and structural features of cholangiocytes in health and disease. Cell Mol Gastroenterol Hepatol 2015, 1:368-380

33. Franchitto A, Onori P, Renzi A, Carpino G, Mancinelli R, Alvaro D, Gaudio E: Recent advances on the mechanisms regulating cholangiocyte proliferation and the significance of the neuroendocrine regulation of cholangiocyte pathophysiology. Ann Transl Med 2013, 1:27

34. Rizvi S, Eaton JE, Gores GJ: Primary sclerosing cholangitis as a premalignant biliary tract disease: surveillance and management. Clin Gastroenterol Hepatol 2015, 13:2152-2165

35. Kolb R, Liu GH, Janowski AM, Sutterwala FS, Zhang W: Inflammasomes in cancer: a double-edged sword. Protein Cell 2014, 5:12-20

36. Wei Q, Mu K, Li T, Zhang Y, Yang Z, Jia X, Zhao W, Huai W, Guo P, Han L: Deregulation of the NLRP3 inflammasome in hepatic parenchymal cells during liver cancer progression. Lab Invest 2014, 94:52-62

37. Wilcz-Villega EM, McClean S, O'Sullivan MA: Mast cell tryptase reduces junctional adhesion molecule-A (JAM-A) expression in intestinal epithelial cells: implications for the mechanisms of barrier dysfunction in irritable bowel syndrome. Am J Gastroenterol 2013, 108:1140-1151

38. Bertiaux-Vandaele N, Youmba SB, Belmonte L, Lecleire S, Antonietti M, Gourcerol G, Leroi AM, Dechelotte P, Menard JF, Ducrotte P, Coeffier M: The expression and the cellular distribution of the tight junction proteins are altered in irritable bowel syndrome patients with differences according to the disease subtype. Am J Gastroenterol 2011, 106:2165-2173

39. Goto Y, Uchida Y, Nomura A, Sakamoto T, Ishii Y, Morishima Y, Masuyama K, Sekizawa K: Dislocation of E-cadherin in the airway epithelium during an antigen-induced asthmatic response. Am J Respir Cell Mol Biol 2000, 23:712-718

40. Rittirsch D, Flierl MA, Nadeau BA, Day DE, Huber-Lang MS, Grailer JJ, Zetoune FS, Andjelkovic AV, Fasano A, Ward PA: Zonulin as prehaptoglobin2 regulates lung permeability and activates the complement system. Am J Physiol Lung Cell Mol Physiol 2013 304:L863-L872

41. Cipriani S, Mencarelli A, Chini MG, Distrutti E, Renga B, Bifulco G, Baldelli F, Donini A, Fiorucci S: The bile acid receptor GPBAR-1 (TGR5) modulates integrity of intestinal barrier and immune response to experimental colitis. PLoS One 2011, 6:e25637

42. Lammers KM, Lu R, Brownley J, Lu B, Gerard C, Thomas K, Rallabhandi P, Shea-Donohue T, Tamiz A, Alkan S, Netzel-Arnett S, Antalis T, Vogel SN, Fasano A: Gliadin induces an increase in intestinal permeability and zonulin release by binding to the chemokine receptor CXCR3. Gastroenterology 2008, 135:194-204.e3

43. Zaki MH, Boyd KL, Vogel P, Kastan MB, Lamkanfi M, Kanneganti TD: The NLRP3 inflammasome protects against loss of epithelial integrity and mortality during experimental colitis. Immunity 2010, 32:379-391

44. Solanas G, Porta-de-la-Riva M, Agusti C, Casagolda D, SanchezAguilera F, Larriba MJ, Pons F, Peiro S, Escriva M, Munoz A, Dunach M, de Herreros AG, Baulida J: E-cadherin controls betacatenin and NF-kappaB transcriptional activity in mesenchymal gene expression. J Cell Sci 2008, 121:2224-2234

45. Shih HC, Shiozawa T, Miyamoto T, Kashima H, Feng YZ, Kurai M, Konishi I: Immunohistochemical expression of E-cadherin and beta-catenin in the normal and malignant human endometrium: an inverse correlation between E-cadherin and nuclear beta-catenin expression. Anticancer Res 2004, 24:3843-3850

46. Eaton JE, Talwalkar JA, Lazaridis KN, Gores GJ, Lindor KD: Pathogenesis of primary sclerosing cholangitis and advances in diagnosis and management. Gastroenterology 2013, 145:521-536

47. Hanada S, Harada M, Koga H, Kawaguchi T, Taniguchi E, Kumashiro R, Ueno T, Ueno Y, Ishii M, Sakisaka S, Sata M: Tumor necrosis factoralpha and interferon-gamma directly impair epithelial barrier function in cultured mouse cholangiocytes. Liver Int 2003, 23:3-11 
48. Sheth P, Delos Santos N, Seth A, LaRusso NF, Rao RK: Lipopolysaccharide disrupts tight junctions in cholangiocyte monolayers by a c-Src-, TLR4-, and LBP-dependent mechanism. Am J Physiol Gastrointest Liver Physiol 2007, 293:G308-G318

49. Fickert P, Fuchsbichler A, Wagner M, Zollner G, Kaser A, Tilg H, Krause R, Lammert F, Langner C, Zatloukal K, Marschall HU, Denk H, Trauner M: Regurgitation of bile acids from leaky bile ducts causes sclerosing cholangitis in Mdr2 (Abcb4) knockout mice. Gastroenterology 2004, 127:261-274

50. Moriyama-Kita M, Endo Y, Yonemura Y, Heizmann CW, Miyamori H, Sato H, Yamamoto E, Sasaki T: S100A4 regulates E-cadherin expression in oral squamous cell carcinoma. Cancer Lett 2005, 230:211-218

51. Yonemura Y, Endou Y, Kimura K, Fushida S, Bandou E, Taniguchi K, Kinoshita K, Ninomiya I, Sugiyama K, Heizmann CW,
Schafer BW, Sasaki T: Inverse expression of S100A4 and E-cadherin is associated with metastatic potential in gastric cancer. Clin Cancer Res 2000, 6:4234-4242

52. Myong NH: Loss of E-cadherin and acquisition of vimentin in epithelial-mesenchymal transition are noble indicators of uterine cervix cancer progression. Korean J Pathol 2012, 46: $341-348$

53. Guarda G, Zenger M, Yazdi AS, Schroder K, Ferrero I, Menu P, Tardivel A, Mattmann C, Tschopp J: Differential expression of NLRP3 among hematopoietic cells. J Immunol 2011, 186: 2529-2534

54. Chen LP, Cai M, Zhang QH, Li ZL, Qian YY, Bai HW, Wei X, Shi BY, Dong JH: Activation of interleukin-6/STAT3 in rat cholangiocyte proliferation induced by lipopolysaccharide. Dig Dis Sci 2009, 54:547-554 